\title{
ORBIT STRUCTURE OF THE EXCEPTIONAL HERMITIAN SYMMETRIC SPACES. II
}

\author{
BY DANIEL DRUCKER ${ }^{1}$
}

Communicated by S. S. Chern, May 7, 1974

This note describes results on the orbit structure of the exceptional hermitian symmetric space $E_{6} / S O(10) \cdot S O(2)$ analogous to those obtained for the space $E_{7} / E_{6} \cdot S O(2)$ in [2].

1. J. Tits' construction of the complex Lie algebra $\mathfrak{F}_{6}$. Let $A$ be the algebra $\mathbf{C} \oplus \mathbf{C}$ with componentwise multiplication, and define the trace of an element of $A$ to be the sum of its two components. As in [2], let $J$ be the 27-dimensional Jordan algebra of hermitian $3 \times 3$ matrices over the complex Cayley numbers. Write $A_{0}$ and $J_{0}$ for the subsets of $A$ and $J$ consisting of elements with zero trace. Also let $\operatorname{Der}(J)$ be the Lie algebra of derivations of $J$ and let $\{L(A)\}(B)=A \circ B$ denote multiplication in $J$. Now define an anticommutative multiplication $[$,$] on the complex vector$ space $g=\left(A_{0} \otimes J_{0}\right)+\operatorname{Der}(J)$ by means of the following rules:

(a) $\left[D, D^{\prime}\right]$ is the usual commutator for $D, D^{\prime} \in \operatorname{Der}(J)$.

(b) $[D, a \otimes A]=a \otimes D(A)$ for $a \in A_{0}, A \in J_{0}$, and $D \in \operatorname{Der}(J)$.

(c) $[a \otimes A, b \otimes B]=1 / 2 \operatorname{Tr}(a b)[L(A), L(B)]$ for $a, b \in \mathrm{A}_{0}$ and $A, B \in J_{0}$.

Then 8 is the complex Lie algebra $\mathfrak{F}_{6}$.

If we put $e=(1,-1) \in A_{0}$, then $A_{0}=\mathbf{C} \cdot e$, so $\mathfrak{g}=\left(\mathbf{C} \cdot e \otimes J_{0}\right)+$ $\operatorname{Der}(J)$, and the multiplication in $g$ is determined by the single rule

$\left[e \otimes A+D, e \otimes A^{\prime}+D^{\prime}\right]=e \otimes\left\{D\left(A^{\prime}\right)-D^{\prime}(A)\right\}+\left[L(A), L\left(A^{\prime}\right)\right]+\left[D, D^{\prime}\right]$

for $A, A^{\prime} \in J_{0}$ and $D, D^{\prime} \in \operatorname{Der}(J)$.

Let $A^{\prime}$ be the set of elements in $A$ of the form $\left(w, w^{*}\right)$, where $w^{*}$

AMS (MOS) subject classifications (1970). Primary 17B25, 17B60, 32M15, 53C35; Secondary 17C40.

Key words and phrases. Hermitian symmetric space, bounded symmetric domain, holomorphic arc component, boundary component.

${ }^{1}$ Research partially supported by NSF GP-38724. 
is the complex conjugate of $w$. Let $J^{\prime}$ be the set of matrices in $J$ whose entries are real Cayley numbers. Choose an element $(i, j, k)$ from the set $\{(1,2,3),(2,3,1),(3,1,2)\}$ and (in the notation of $[2, \S 5])$ let $J^{\prime \prime}$ be the subset of $J$ whose entries are of the form $\xi_{i} E_{i}+\xi_{j} E_{j}+\xi_{k} E_{k}+F_{i}\left\{x_{i}\right\}+$ $F_{j}\left\{x_{j}\right\}+F_{k}\left\{x_{k}\right\}$, where $\xi_{i}, \xi_{j}, \xi_{k}$ are real numbers and $\sqrt{-1} x_{i}, \sqrt{-1} x_{j}$, $x_{k}$ are real Cayley numbers. If we substitute $A^{\prime}$ and $J^{\prime}$ for $A$ and $J$ in the above construction, we obtain a compact real form $\mathfrak{g}_{c}$ of $\mathfrak{g}$. If instead we substitute $A^{\prime}$ and $J^{\prime \prime}$, we obtain a noncompact real form $\mathfrak{g}_{0}$ of g with Cartan index -14 .

All of the above results come from [7].

2. Conventions. Put $X_{c}=E_{6} / S O(10) \cdot S O(2)$ and let $X_{0}$ be the noncompact dual of $X_{c}$. Define $\mathfrak{t}$ and $\mathrm{m}_{0}$ as in $[2, \S 1]$, write $X_{0}=$ $G_{0} / K$ and $X_{c}=G_{c} / K$ as in $[2, \S 2]$, and define $\mathrm{m}^{+}, \mathrm{m}^{-}, \xi, \Omega$, and $f_{u}$ (for $u \in \mathfrak{m}^{+}$) as in $[2, \S \S 3,4]$.

Let $K$ denote the subspace of $J$ consisting of elements of the form $F_{i}\left\{x_{i}\right\}+F_{j}\left\{x_{j}\right\}$, where $x_{i}$ and $x_{j}$ are complex Cayley numbers.

3. Realization of $X_{0}$ as a bounded symmetric domain. Using the construction of $\mathfrak{g}_{0}$ and $\mathfrak{g}_{c}$ in $\S 1$, we find that $\mathrm{m}^{+}$and $\mathrm{m}^{-}$are isomorphic to $K$. If we identify $\mathrm{m}^{+}$and $\mathrm{m}^{-}$with $K$, then for each $u \in \mathrm{m}^{+}=$ $K, f_{u}$ can be viewed as the endomorphism of $\mathrm{m}^{-}=K$ defined by

$$
f_{u}=2\left\{L\left(u \circ u^{*}\right)-\left[L(u), L\left(u^{*}\right)\right]\right\}
$$

Hence by Langlands' theorem (cf. [2, §4]), we obtain

THEOREM 1. $\Omega=\left\{u \in K: L\left(u \circ u^{*}\right)-\left[L(u), L\left(u^{*}\right)\right]<I\right\}$.

As in [2], this description can be compared with those obtained by M. Koecher [6] and M. Ise [4], [5], who used different methods. Renumbering so that the choices of $(i, j, k)$ coincide, we obtain

THEOREM 2. The three descriptions of $\Omega$ are identical as point sets.

4. Notations. If $c$ and $d$ are nonnegative integers such that $0 \leqslant$ $c+d \leqslant 16$, let $K(c, d)$ denote the set of matrices $u$ in $K$ such that $f_{u}$ has $c$ eigenvalues $<2$ and $d$ eigenvalues $>2$ (hence $16-c-d$ eigenvalues $=2)$. Then $\Omega=K(16,0)$ by Theorem 1 .

Let $\left\{e_{0}, e_{1}, \cdots, e_{7}\right\}$ be the usual basis for the complex Cayley numbers. Define another basis $\left\{e_{0}^{\prime}, e_{1}^{\prime}, \cdots, e_{7}^{\prime}\right\}$ by 
$e_{2 t}^{\prime}=1 / 2\left(\sqrt{-1} e_{t}+e_{7-t}\right) \quad$ and $\quad e_{2 t+1}^{\prime}=1 / 2\left(-\sqrt{-1} e_{t}+e_{7-t}\right), \quad 0 \leqslant t \leqslant 3$.

Let $\Delta$ be the set of matrices of $K$ of the form $F_{i}\left\{r e_{0}^{\prime}+s e_{1}^{\prime}\right\}$, where $r$ and $s$ are real numbers. If $a$ and $b$ are nonnegative integcrs with $0 \leqslant$ $a+b \leqslant 2$, let $\Delta(a, b)$ denote the $\operatorname{Ad}(K)$-orbit of the set of matrices $F_{i}\left\{r e_{0}^{\prime}+s e_{1}^{\prime}\right\}$ in $\Delta$ such that $a$ of the numbers $r^{2}, s^{2}$ are $<1$ and $b$ of them are $>1$.

5. The $G_{0}$-orbit structure of $X_{c}=E_{6} / S O(10) \cdot S O(2)$. The following result is proved by means of general theory from [9] and a study of the eigenvalues of $f_{u}$ for $u \in K$.

THEOREM 3. The pullbacks under $\xi$ of the $G_{0}$-orbits on $X_{c}$ are the sets $\Delta(a, b)$, where $a$ and $b$ are nonnegative integers such that $0 \leqslant a+$ $b \leqslant 2$. These sets can be described in terms of the eigenvalues of $f_{u}, u \in K$, as follows:

$$
\begin{aligned}
& \Delta(2,0)=K(16,0), \\
& \Delta(0,2)=K(0,16) \cup K(4,12) \cup K(8,8) \cup K(0,12) \cup K(4,8) \cup K(0,8), \\
& \Delta(1,1)=K(15,1) \cup K(9,7) \cup K(5,11) \cup K(5,7) \cup K(9,1) \cup K(5,1), \\
& \Delta(1,0)=K(15,0), \\
& \Delta(0,1)=K(8,7) \cup K(4,11) \cup K(4,7), \text { and } \\
& \Delta(0,0)=K(8,0) .
\end{aligned}
$$

Let $S(a, b)$ denote the $G_{0}$-orbit on $X_{c}$ whose pullback under $\xi$ is $\Delta(a, b)$. Then

(a) The open $G_{0}$-orbits on $X_{c}$ are $X_{0}=S(2,0), S(1,1)$, and $S(0,2)$.

(b) The topological boundaries in $X_{c}$ of the open orbits $S(2,0)$, $S(1,1)$, and $S(0,2)$ are $S(1,0) \cup S(0,0), S(1,0) \cup S(0,1) \cup S(0,0)$, and $S(0,1) \cup S(0,0)$ respectively.

(c) $S(0,0)$ is the Bergman-Silov boundary of $X_{0}$ in $X_{c}$, the unique closed $G_{0}$-orbit on $X_{c}$.

(d) $S\left(a^{\prime}, b^{\prime}\right)$ is in the closure of $S(a, b)$ if and only if $a^{\prime} \leqslant a$ and $b^{\prime} \leqslant b$.

(Part (d) of Theorem 3 in [2] is incorrect. It should be identical to part (d) of the above theorem.) 
6. Holomorphic arc components. Theorem 4 below is proved by means of results from [9] and the eigenvalue analysis mentioned in $\S 5$. The determination of the rank 1 holomorphic arc components necessitates some computations involving the orbit structure of the rank 1 hermitian symmetric space $S U(6) / S\left(U_{1} \times U_{5}\right)$ (notation as in [3, p. 354]), whose noncompact dual has a bounded realization as the open unit ball in $\mathbf{C}^{5}$.

THEOREM 4. Let $a$ and $b$ be nonnegative integers with $0 \leqslant a+b \leqslant$ 2. Then the holomorphic arc components of the $G_{0}$-orbit $S(a, b)$ are symmetric spaces of rank $a+b$ whose pullbacks under $\xi$ are the sets $\operatorname{Ad}(K) \cdot$ $C(a, b), k \in K$, where the subset $C(a, b)$ of $\mathrm{m}^{+}=K$ is described for each choice of $a$ and $b$ as follows: Choose $(i, j, k)$ as in $\S 1$. Then

$$
\begin{aligned}
& C(0,0)=\left\{-F_{i}\left\{e_{7}\right\}\right\}, \\
& C(1,0)=\left\{F_{i}\left\{-e_{0}^{\prime}+z_{1} e_{1}^{\prime}\right\}+F_{j}\left\{z_{2} e_{0}^{\prime}+z_{3} e_{2}^{\prime}+z_{4} e_{4}^{\prime}+z_{5} e_{6}^{\prime}\right\}: \sum_{m=1}^{5}\left|z_{m}\right|^{2}<1\right\}, \\
& C(0,1)=\left\{F_{i}\left\{-e_{0}^{\prime}+z_{1} e_{1}^{\prime}\right\}+F_{j}\left\{z_{2} e_{0}^{\prime}+z_{3} e_{2}^{\prime}+z_{4} e_{4}^{\prime}+z_{5} e_{6}^{\prime}\right\}: \sum_{m=1}^{5}\left|z_{m}\right|^{2}>1\right\}, \\
& C(a, b)=\Delta(a, b) \text { when } a+b=2 .
\end{aligned}
$$

In particular, the boundary components of $X_{0}$ have pullbacks $\operatorname{Ad}(k) \cdot$ $C(a, 0)$ where $k \in K$ and $a=0$ or 1 .

Details and complete proofs for the results in this note and in the previous one [2] will appear elsewhere.

\section{REFERENCES}

1. D. Drucker, Nonassociative algebras and hermitian symmetric spaces, Doctoral Dissertation, University of California, Berkeley, Calif., 1973.

2. - Orbit structure of the exceptional hermitian symmetric spaces. I, Bull. Amer. Math. Soc. 80 (1974), 285-289.

3. S. Helgason, Differential geometry and symmetric spaces, Pure and Appl. Math., vol. 12, Academic Press, New York, 1962. MR 26 \#2986.

4. M. Ise, Realization of irreducible bounded symmetric domain of type (V), Proc. Japan Acad. 45 (1969), 233-237. MR 40 \#2905.

5. M. Ise, On canonical realizations of bounded symmetric domains as matrixspaces, Nagoya Math. J. 42 (1971), 115-133. MR 44 \#7478.

6. M. Koecher, An elementary approach to bounded symmetric domains (with additions), Rice University, Houston, Texas, 1969. MR 41 \#5652. 
7. J. Tits, Algèbres alternatives, algèbres de Jordan et algèbres de Lie exceptionelles (announcement), 1963.

8. — Algèbres alternatives, algèbres de Jordan et algèbres de Lie exception-

elles. I. Construction, Nederl. Akad. Wetensch. Proc. Ser. A $69=$ Indag. Math. 28 (1966), 223-237. MR 36 \#2658.

9. J. A. Wolf, Fine structure of hermitian symmetric spaces, Geometry and Analysis of Symmetric Spaces, Marcel Dekker, New York, 1972.

DEPARTMENT OF MATHEMATICS, UNIVERSITY OF WASHINGTON, SEATTLE, WASHINGTON 98195 